\title{
CONDUTA FONOAUDIOLÓGICA E AVALIAÇÃO ESTROBOSCÓPICA NO DIAGNÓSTICO DIFERENCIAL DO CISTO
}

\section{Speech therapy and stroboscopy evaluation in cyst differential diagnosis}

\author{
Silvana da Gama Pastana ${ }^{(1)}$, Esinéa Gomes ${ }^{(2)}$, Luciana Castro ${ }^{(3)}$
}

\begin{abstract}
RESUMO
Objetivo: descrever o trabalho fonoaudiológico, após diagnóstico diferencial do cisto intracordal, por meio do exame de videolaringoestroboscopia associado à fonação inspiratória. Métodos: relato de caso, baseado em dados de anamnese, da avaliação clínica fonoaudiológica, do exame videolaringoestroboscópico e conduta terapêutica específica. Resultados: identificação do cisto intracordal em prega vocal direita com alterações encontradas no exame laringológico, compatível à avaliação clínica fonoaudiológica. Verificou-se que, após conduta direcionada, a paciente obteve melhora na vibração e amplitude da onda mucosa da prega vocal direita, com manutenção de períodos de estabilidade vocal. Conclusão: a eficiência dos procedimentos depende diretamente das avaliações e diagnósticos precisos dos profissionais envolvidos, o que permite uma conduta direcionada ao caso e, menor tempo de tratamento.
\end{abstract}

DESCRITORES: Distúrbios da Voz; Vibração; Fonação; Cistos; Diagnóstico Diferencial

\section{INTRODUÇÃO}

Os aspectos envolvidos na fonação são: anátomofisiológicos, psicossociais e ambientais ${ }^{1}$. Já a qualidade vocal depende das características de todo o trato vocal. Sendo assim, qualquer alteração poderá desencadear uma disfonia, que dependendo de determinados fatores, poderá ser em maior ou menor grau. Na avaliação dos aspectos anátomo-fisiológicos existem instrumentos que identificam várias alterações.

Na semiologia otorrinolaringológica, há exames que oferecem uma análise eficiente do comportamento

(1) Fonoaudióloga da Prefeitura do Rio de Janeiro e da Universidade do Estado do Rio de Janeiro; Especialização em Voz pelo CEFAC - Saúde e Educação.

(2) Fonoaudióloga; Especialização em Voz pelo CEFAC - Saúde e Educação.

(3) Fonoaudióloga Docente da Faculdade de Medicina, Curso de Fonoaudiologia da Universidade Federal do Rio de Janeiro; Mestre em Tecnologia Educacional pela Universidade Federal do Rio de Janeiro. vocal com ótima visualização das estruturas laríngeas. Dentre estas, a Estroboscopia laríngea se destaca pelo detalhamento do movimento vibratório da mucosa 2,3, permitindo um diagnóstico laringológico com muito maior precisão ${ }^{4}$. Esta consiste em uma imagem ilusória de câmera lenta, tornando o padrão de vibração das pregas vocais avaliável a olho nu. A utilização de uma luz estroboscópica permite um efeito relentado. Esta luz é descontínua, constituída por uma sucessão de flashes muito curtos ${ }^{5}$. A composição do ciclo completo é obtida pela soma seqüencial de pequenos trechos iluminados de ciclos vibratórios sucessivos.

A laringoestroboscopia possibilita avaliação detalhada da qualidade vibratória das pregas vocais e, os aspectos a serem observados são: fechamento glótico; mobilidade das pregas vocais; nível vertical das pregas vocais; simetria; peridiocidade; amplitude de vibração; onda de mucosa; segmentos fracos; fases do ciclo; atividades supraglóticas e freqüência fundamental ${ }^{2,3,6}$.

O revestimento mucoso das pregas vocais pode abrigar inúmeras patologias e influencia diretamente 
na qualidade vocal. Dentre essas patologias, incluise o cisto de prega vocal ou intracordal que pode ser definido como uma lesão benigna de origem epitelial, que apresenta retenção líquida, pastosa ou mesmo sólida, revestido por epitélio pluriestratificado escamoso e queratinizado ${ }^{7}$. Corresponde a uma cavidade fechada, localizado profundamente no interior da prega vocal, em geral na camada superficial da lâmina própria, geralmente sem aderência ao epitélio e com ou sem aderência ao ligamento vocal ${ }^{8,9}$, localiza-se em terço médio da prega vocal, geralmente unilateral e associado a um entrelaçado vascular ou vasculodisgenesia ${ }^{2,8-10}$. Pode permanecer assintomático ou provocar disfonia de graus variados, por redução da elasticidade da túnica mucosa ou pela presença do abaulamento que provoca na prega vocal ${ }^{1}$.

A fonação inspiratória, reversa ou inalatória é produzida durante a inspiração e é facilmente observada no soluço, choro, riso e engasgo ${ }^{11}$. Consiste na emissão de um som durante $\mathrm{o}$ ato inspiratório fazendo com que ocorra o relaxamento do vestíbulo laríngeo, promove o movimento da mucosa a favor da força da gravidade. Tem sido utilizada como técnica terapêutica na clínica fonoaudiológica ${ }^{2,3} \mathrm{e}$ ainda, como recurso no diagnóstico diferencial das lesões de massa, auxiliando na identificação de cistos de pregas vocais ${ }^{12}$.

A avaliação fonoaudiológica contribui para definir a importância do uso da voz na etiologia e na manutenção da disfonia, oferece dados para um processo de diagnóstico diferencial e ainda, identifica comportamentos, personalidade e motivação na proposta de uma reabilitação vocal. A avaliação detalhada e precisa é a primeira etapa do tratamento e leva a um plano de reabilitação específico e direcionado aos sintomas do paciente. A avaliação do paciente disfônico é multiprofissional e envolve, necessariamente, um diagnóstico médico e uma avaliação fonoaudiológica ${ }^{3}$. Tal parceria possibilita coletar dados relevantes das avaliações e favorecer o direcionamento da conduta terapêutica.

Portanto, o objetivo deste trabalho é descrever a conduta fonoaudiológica, baseada em dados das avaliações perceptivo-auditiva e exame laringológico específico, no diagnóstico do cisto.

\section{MÉTODOS}

Apresentação de relato de caso, baseado nas informações coletadas por meio de anamnese e das avaliações do comportamento vocal ${ }^{2,3}$ da paciente e dos dados das avaliações laringológicas que constam nos exames de videolaringoestroboscopia, realizada por médico otorrinolaringologista.

Após realizada a coleta dos dados em anamnese, a paciente foi submetida à avaliação do comportamento vocal e a análise perceptivoauditiva ${ }^{2,3}$. Após realização das avaliações fonoaudiológicas, a paciente foi reencaminhada a uma nova avaliação otorrinolaringológica. Nesta, o exame de eleição foi a videolaringoestroboscopia associada à fonação inspiratória, tal manobra foi utilizada a pedido da terapeuta ao médico otorrinolaringologista.

A conduta fonoaudiológica foi baseada após diagnóstico diferencial do exame de videolarin-goestroboscopia e consistiu na aplicação de técnicas direcionadas ao caso. Além de todas as orientações sobre o uso profissional da voz e de higiene vocal com objetivo de eliminar e/ ou minimizar os mecanismos compensatórios negativos, a paciente foi esclarecida de toda a fisiologia normal do aparelho fonador e do uso adequado da voz e ainda, de todas as alterações apresentadas nas avaliações. Os mecanismos de compensação foram retirados para possibilitar os adequados ajustes posteriores. A paciente utilizava as bandas ventriculares por compensação extrínseca como meio de manter a produção de sua voz, com isso, gerava excessiva tensão no pescoço e cintura escapular. As técnicas utilizadas foram:

- a fonação inspiratória que pela fisiologia reversa, evita que as pregas ventriculares entrem em atividade, aproximando as pregas vocais com menor impacto, favorecendo a onda mucosa;

- a vibração de língua com cabeça rodada para a direita e levemente abaixada, permitiu dar maior flexibilidade à prega vocal direita e promoveu melhora de sua amplitude;

- o exercício do som nasal $/ \mathrm{m} /$ é uma técnica bastante utilizada para melhorar a coaptação glótica, reduzir o foco de ressonância laringofaríngea, proporcionar ampliação do trato vocal sem gerar tensão favorecendo à projeção vocal e, aumentar os tempos máximos de fonação;

- o /b/ prolongado foi utilizado para melhorar a coaptação glótica sem impacto, flexibilizar a posição vertical da laringe no pescoço, aumentar a onda mucosa e por conseqüência melhorar a qualidade vibratória;

- a voz salmodiada em curva melódica ascendente e descendente promoveu suavidade à emissão e aumento da resistência e extensão vocal.

Após 12 sessões, a paciente foi novamente encaminhada à avaliação laringológica, fazendo um segundo exame de videolaringoestroboscopia, cuja observação dos dados foram: melhora de vibração e da amplitude de prega vocal direita e diminuição da atividade das pregas vestibulares.

A presente pesquisa foi avaliada e aprovada pelo Comitê de Ética em Pesquisa do CEFAC - Saúde e Educação, sob o n¹25/06, considerada sem risco e com necessidade do consentimento livre e esclarecido. 


\section{RESULTADOS}

Descrição do Caso: paciente do sexo feminino, 37 anos, professora, com queixa de fadiga vocal e afonia no final do dia. Referia rouquidão desde criança, com conseqüente piora após ingressar no magistério, sendo que, a queixa de afonia apareceu quando houve um aumento da demanda vocal pelo exercício da profissão. Após várias crises de afonia, procurou um médico, no que foi indicada à avaliação de videolaringoscopia, num total de cinco exames, todos com diagnóstico de fenda glótica e, em alguns, havia a observação de formação de ectasia vascular.

$\mathrm{Na}$ análise perceptivo-auditiva ${ }^{2,3}$, foram observadas as seguintes alterações: aspereza, rouquidão, leve soprosidade, diplofonia; pitch agudo; loudness reduzida, aumento da tensão em cintura escapular associada a quebras de sonoridade durante a fala espontânea e ressonância laringofaríngea, laringe em posição alta no pescoço com grande esforço à fonação; relação s/z indicativa de falta de coaptação glótica.

$\mathrm{Na}$ avaliação otorrinolaringológica, foram obtidos os seguintes dados: pequena amplitude da vibração; imagem nodular no terço médio de prega vocal direita, sugestiva de cisto intracordal com alteração de linearidade da borda livre, sendo esta área hipodinâmica; vasculodisgenesia especialmente em terço médio de prega vocal direita; fechamento inconsistente em fenda semi-ampulheta; hiperemia de aritenóides; atividade aumentada de bandas ventriculares.

Após conduta direcionada, a paciente apresentou resultado satisfatório em 12 sessões. $\mathrm{Na}$ análise perceptivo-auditiva foi observada melhora da qualidade vocal com aumento dos tempos máximos de fonação e da extensão vocal, maior resistência vocal, mantendo rouquidão de grau leve, emissão mais suave e menor tensão na região do pescoço e cintura escapular à fonação. Na segunda avaliação laringológica, foi observada melhora da vibração e da amplitude de prega vocal direita e ainda, diminuição da atividade de pregas ventriculares.

Apesar do resultado satisfatório, a paciente apresenta, em alguns momentos, instabilidade vocal, com qualidade áspera moderada pela rigidez de mucosa, por vezes diplofônica e curva melódica limitada e ainda, por apresentar outras alterações gástricas e respiratórias. A presença da patologia mantém a irregularidade vibratória e assimetria de fase. Deste modo, foi orientada à clínica Otorrinolaringológica para avaliação de uma possível conduta cirúrgica. No que deverá retornar após cirurgia à clinica fonoaudiológica para reavaliação do comportamento vocal.

A paciente encontra-se atualmente em plena atividade e relatou não mais ter crises de afonia e refere estar satisfeita com os resultados obtidos em terapia.

\section{DISCUSSÃO}

A reabilitação vocal, atualmente, dispõe de inúmeros recursos que podem favorecer ao indivíduo com disfonia, resgatar suas qualidades vocais, ou promover, dentro das possibilidades anatomo-fisiológicas, condições que favoreçam aos ajustes vocais mais adequados para cada caso. Através do conhecimento da anatomia e fisiologia e, especialmente, pelo desenvolvimento de aparelhos que possibilitam a visualização e funcionamento das estruturas laríngeas, permitem esclarecer e, em muitos casos, definir patologias que sem uma semiologia avançada, dificultaria a conclusão diagnóstica e o tratamento específico.

A avaliação perceptivo-auditiva é a avaliação clássica da qualidade vocal, tradicional na rotina clínica fonoaudiológica na área de voz e, apesar de subjetiva, é considerada bastante confiável e eficaz na correlação dos dados com os exames objetivos do paciente. Os achados desta avaliação permitiram sugerir que se tratava de uma alteração estrutural mínima, já que os dados da anamnese e a qualidade vocal da paciente apontavam para esta hipótese diagnóstica. Diante disso, a paciente foi encaminhada a uma nova reavaliação laringológica que consistiu de exame de videolaringoestroboscopia com o recurso da fonação inspiratória, recurso este sugerido pela terapeuta, realizada por médico otorrinolaringologista e com diagnóstico final de cisto intracordal em prega vocal direita.

O cisto intracordal é uma alteração estrutural mínima de cobertura de prega vocal, de origem congênita e caráter benigno, que pode se manifestar em qualquer idade, principalmente com o uso intenso e abusivo da voz. Considerado de incidência comum na faixa etária de dez a 80 anos, predominantemente no sexo feminino e, mais freqüentemente associado à vasculodisgenesia 7,13-16. A sintomatologia é mais evidenciada na faixa de 31 a 40 anos $^{7}$, sendo mais observada em profissionais da voz ${ }^{17}$, que pela demanda vocal, são suscetíveis a desenvolver alterações vocais e assim gerar disfonias 17-20. Seu diagnóstico muitas vezes é confundido com outras patologias, que em geral apresenta-se como um espessamento inflamatório da prega vocal, acompanhado de hiperemia de mucosa adjacente $e$ de ectasia capilar ${ }^{21}$. Quando não utilizados os meios específicos que permitam um diagnóstico correto, poderá levar a condutas não direcionadas ${ }^{22}$, no que implicará em resultados ineficientes ao quadro apresentado pelo paciente. Atualmente, já é possível contar com recursos que resultam em uma melhor precisão diagnóstica, como no caso do exame de videolaringoestroboscopia que detalha o movimento vibratório das pregas vocaise, permite visualização de suas irregularidades vibratórias ${ }^{2-4}$, sendo considerado 
o melhor método para identificação do cisto intracordal ${ }^{23,24}$ e, sua associação com a fonação inspiratória, vem acrescentar valioso recurso no diagnóstico das lesões benignas de pregas vocais ${ }^{7,12}$. Esta manobra permite a visualização do ligamento vocal pelo desprendimento da camada superficial, o que poderá revelar área de rigidez com diminuição ou ausência da onda mucosa ${ }^{25}$, que em presença de lesões mais rígidas não ocorre a expansão da camada superficial da lâmina própria, limitando ou impedindo a visualização da linha do ligamento vocal ${ }^{12}$ e que possibilita, na maioria das vezes, definir, identificar e localizar as lesões de massa e suas características específicas, principalmente na identificação dos cistos intracordais ${ }^{7,12,26}$ e, desse modo, contribui para um diagnóstico diferencial e conseqüentemente, leva à conduta direcionada com melhor qualidade e menor tempo de tratamento.

Os achados da avaliação percetivo-auditiva foram correlacionados aos achados do exame laringológico da paciente ${ }^{27}$, demonstrando a importância da análise perceptiva no direcionamento da conduta fonoaudiológica. Os aspectos vocais são descritos na literatura e são utilizados para qualificar a voz na avaliação perceptivo-auditiva ${ }^{1-3,28}$

As alterações mais relevantes da qualidade vocal foram: a aspereza, que pode correlacionar-se com uma rigidez de mucosa com redução ou ausência da onda mucosa que pode sugerir alterações congênitas na arquitetura histológica das pregas vocais, como sulcos e cistos intracordais, nas leucoplasias, nas retrações cicatriciais pós cirúrgicas e nas iatrogenias; a rouquidão, que sugere presença de irregularidade vibratória da mucosa de pregas vocais durante a fonação e, que pode ser justificada pela presença de fenda isolada ou associada à patologia na mucosa vibrante das pregas vocais como pólipos, nódulos, hiperemia e edemas, vasodilatação, neoplasias e gripes; a diplofonia que é a produção vocal simultânea de dois tons, que pode ocorrer por duas freqüências de uma prega vocal vibrando diferente da outra, vibração de pregas vocais e pregas ventriculares e das estruturas ariepiglótica; a soprosidade, que corresponde à presença de ruído de fundo audível, o que demonstra fechamento glótico incompleto por apresentação de fenda, pode estar relacionada às disfonias hipocinéticas, quadros de fadiga vocal, inadaptação fônica e casos neurológicos. Há estudos que comprovam a relação de determinados tipos de fendas na associação com os nódulos e as alterações estruturais mínimas ${ }^{29,30}$.

Todos os achados da análise da qualidade vocal foram correlacionados ao exame videolaringoestroboscópico. As alterações relevantes neste exame foram: vibração restrita e rigidez de mucosa com alteração de amplitude e assimetria de fase em prega vocal direita com restrição do movimento muco ondulatório pela presença de alteração de cobertura de pregas vocais, no caso em questão, o cisto intracordal, o que justifica a aspereza; vasculodisgenesia em prega vocal direita principalmente em terço médio que, segundo a literatura, é forte indicador da presença de alterações estruturais mínimas $14,15,21$, especialmente do cisto intracordal ${ }^{7,13,16}$; a atividade de bandas ventriculares aumentada, configura em movimento compensatório ${ }^{31}$ da musculatura extrínseca, pela ineficiência glótica e restrição do movimento vibratório ${ }^{25}$; apresentou também hiperemia de aritenóides que, de acordo com estudos, está associada ao refluxo gastroesofágico ${ }^{32,33}$, quadro também relatado pela paciente; e fenda, que é justificada pela presença de abaulamento em terço médio em decorrência da alteração estrutural, o que impede uma coaptação adequada das pregas vocais $^{9,16}$.

A conduta fonoaudiológica foi direcionada a partir do diagnóstico diferencial, o que permitiu a utilização de técnicas apropriadas às alterações encontradas. Foram priorizadas as técnicas que favorecessem os ajustes vocais adequados e eliminação dos ajustes compensatórios.

A vibração de língua é considerada uma das técnicas mais efetivas para promover a soltura de onda mucosa pelo deslizar da cobertura mucosa das pregas vocais sobre seu corpo, ativando a circulação sanguínea periférica e massageando a mucosa que reveste as pregas vocais ${ }^{1}$ favorece a flexibilidade e amplitude vibratória, permitindo uma emissão normotensa e equilibrada em ressonância com produção mais estável e efeito positivo imediato, com melhora do ajuste das pregas vocais e conseqüentemente da qualidade vocal ${ }^{1,2,28,34-36}$. Sua associação com a postura de cabeça rodada e levemente abaixada, favorece a prega vocal mais alterada e possibilita seu nivelamento ${ }^{28,34}$, além disso, a variação com escalas ascendentes e descendentes permite um condicionamento muscular laríngeo com conseqüente aumento da resistência vocal.

$\mathrm{O} / \mathrm{b} /$ prolongado foi utilizado para alterar a posição vertical da laringe, já que a paciente demonstrava posição de laringe alta. Este recurso possibilita uma posição mais baixa no pescoço ${ }^{37}$, com melhora da coaptação glótica ${ }^{1,34}$ e aumento dos tempos máximos de fonação ${ }^{38}$, favorece a assimetria de fase vibratória entre as pregas vocais ${ }^{28}$ e aumento da amplitude de vibração de mucosa ${ }^{39}$.

A técnica de som nasal com utilização do $/ \mathrm{m} /$ permite percepção das vibrações na face, facilitando a projeção vocal, foi utilizada para suavizar a emissão e reduzir o foco de ressonância laringo-faríngea 
apresentada pela paciente, favorece à coaptação glótica com aumento dos tempos máximos de fonação ${ }^{2,28,34}$.

A voz salmodiada permite coaptação de pregas vocais de modo suave, desse modo, o foco de ressonância distribui-se projetando melhor a voz no ambiente ${ }^{34}$. Foi utilizada para dar maior estabilidade à emissão suave, favorecer a manutenção da ressonância trabalhada nos exercícios anteriores em segmentos de fala maiores ${ }^{28}$, com o objetivo de aumentar a estabilidade suave da fala espontânea da paciente.

A fonação inspiratória foi adotada como forma de inibir o movimento compensatório utilizado pela medialização de pregas ventriculares. Na literatura há estudos que comprovam a efetividade da fonação inspiratória como recurso terapêutico por apresentar freqüência fundamental mais elevada, baixa variação de intensidade e menor contato de pregas vocais ${ }^{40}$. $\mathrm{O}$ ar inspiratório afasta as pregas ventriculares e participa da vibração mucosa das pregas vocais ${ }^{28,34}$.
Todos os exercícios empregados foram pré-selecionados através de provas terapêuticas ${ }^{41}$, que consistem na escolha das abordagens a serem utilizadas na terapia, a fim de não trazerem desconforto à paciente.

\section{CONCLUSÃO}

O presente estudo permitiu concluir que, os procedimentos fonoaudiológicos foram eficientes para este caso, e que, obteve-se o resultado esperado através do direcionamento da conduta terapêutica, possibilitado por avaliações e diagnósticos precisos por parte dos profissionais envolvidos, com o objetivo de proporcionar à paciente, menor tempo de tratamento, levando-se em conta, os limites anatômicos e fisiológicos que o indivíduo apresenta e, quando necessário, reencaminhar à clínica Otorrinolaringológica para uma possível conduta cirúrgica.

\section{ABSTRACT}

Purpose: to describe the voice therapy after a cyst differential diagnosis by means of videostrobolaryngoscopy associated with inspiratory phonation. Methods: vase study based on clinical evaluation anamnesis data of phonoaudiologic, videostroboscopic examination, and specific therapeutic conduct. Results: cyst identification in right vocal fold and alterations found in the laryngological examination which were compatible with phonoaudiologic clinical evaluation. After guides speech therapy, we verified evolution in vibration and amplitude of the mucous wave of the right vocal fold, with maintenance of periods as for vocal stability. Conclusion: the efficiency of the preceding methods directly depends on the precise evaluations and diagnosis, of the involved professionals, which allows for a conduct guided to the case and less treatment time.

KEYWORDS: Voice Disorders; Vibration; Phonation; Cysts; Diagnosis, Differential

\section{REFERÊNCIAS}

1.Pinho SMR. Fundamentos em fonoaudiologia: tratando os distúrbios da voz. Rio de Janeiro: GuanabaraKoogan; 2003. p. 3-39.

2.Behlau M, Pontes P. Avaliação e tratamento das disfonias. São Paulo: Lovise; 1995. p. 143-66.

3.Behlau M, Madazio G, Feijó D, Pontes P. Avaliação de voz. In: Behlau M. Voz: o livro do especialista. Rio de Janeiro: Revinter; 2001. p. 83-172.

4.Fulgêncio MST, Meneses JW, Teixeira AS. Laringoestroboscopia. Rev Bras Otorrinolaringol. 1986; 52(2):11-5.

5.Le Huche F, Allali A. A voz: patologia vocal de origem funcional. Porto Alegre: Artmed; 2005. p. 36-7.

6.Avellaneda CE, Martinez LM. Nuevas alternativas em estroboscopia laringea: computarizada y/o digital. [documento online]. Disponível em: URL: http:// www.encolombia.com/otorrino28200-nuevasalter.htm. 7.Steffen N, Moschetti MB, Zaffari RT. Cistos de pregas vocais: análise de 96 casos. Rev Bras Otorrinolaringol. 1995: 61(3):179-86.

8.Pontes PAL, Gadelha MEC, Gonçalves MIR. Alterações estruturais mínimas da laringe. In: Pinho SMR. Fundamentos em fonoaudiologia: tratando os distúrbios da voz. Rio de Janeiro: GuanabaraKoogan; 2003. p. 67-73.

9.Behlau M, Azevedo R, Pontes P, Brasil O. Disfonias funcionais. In: Behlau M. Voz: o livro do especialista. Rio de Janeiro: Revinter; 2001. p. 247-81.

10. Le Huche F, Allali A. A voz: patologia vocal de origem orgânica. Porto Alegre: Artmed; 2005. p. 80-9. 
11. Grau SM, Robb MP, Cacace AT. Acoustic correlates of inspiratory phonation during infant cry. $\mathrm{J}$ Speech Hear Res. 1995; 38(2):373-81.

12. Lopes MV, Behlau M, Brasil OC, Andrade D. A utilização da fonação inspiratória na caracterização das lesões benignas da laringe. Rev Bras Otorrinolaringol. 2000; 66(5):512-8.

13. Bouchayer M, Cornut G. Microsurgery for lesions benign of the vocal folds. Ear Nose Throat J. 1998; 67:446-66.

14. Pontes PAL, De Biase NG, Behlau M. Vascular characteristics of the vocal fold cover in control larynges and larynges with benign lesions. Phonoscope. 1999; 2(30):129-35.

15. Mello ECM, Brito LL, Brasil OCL, Behlau M, Melo DM. Incidências de lesões laríngeas não neoplásicas em pacientes com queixas vocais. Rev Bras Otorrinolaringol. 2001; 67(6):788-94.

16. D'Avila JS, Sennes LU, Tsuji DH. Estudo comparativo da microvascularização das pregas vocais humanas acometidas por cisto e reação nodular contra-lateral sob endoscopia rígida. Rev Bras Otorrinolaringol. 2003; 69(2):166-73.

17. Urrutikoetxea A, Ispizua A, Matellanes F. Pathologie vocale chez lês professeurs: une étude vidéolaryngo-stroboscopique de 1.046 professeurs. Rev Laryngol Otol Rhinol. 1995; 116(4):255-62.

18. Silva TE, Rabuske PA, Bernz MCN, Haas LJ, Nemetz MA. Alterações estruturais mínimas múltiplas e sincrônicas de laringe em paciente profissional da voz falada: relato de caso. Rev Bras Otorrinolaringol.1998; 64(6):636-8.

19. Fuess VLR, Lorenz MC. Disfonia em professores do ensino municipal: prevalência e fatores de risco. Rev Bras Otorrinolaringol. 2003; 69(6):807-12.

20. Almeida SIC. Cisto submucoso de prega vocal: manifestação clínica de laringo-patia relacionada ao trabalho. Arq Otorrinolaringol. 2005; 9(3):242-8.

21. Tsuji DH, Chung D. Alterações estruturais mínimas das pregas vocais. In: Pinho SMR, Tsuji $\mathrm{DH}$, Bohadana SC. Fundamentos em laringologia e voz. Rio de Janeiro: Revinter; 2006. p. 53-67.

22. Costa EMF, Sant'Anna GD. Erro diagnóstico em disfonia orgânico-funcional: uma entidade freqüente. In: Behlau M, organizador. O melhor que vi e ouvi: atualização em laringe e voz. Rio de Janeiro: Revinter; 1998. p. 65-9.

23. Sataloff RT, Spiegel JR, Hawkshaw M, Rosen DC. Vocal fold cysts. Ent J. 1993. p. 328.

24. Shohet JA, Courey MS, Scott MA, Ossoff RH. Value of videostroboscopic parameters in differentiating true vocal fold cysts from polyps. Laryngoscope. 1996;106:19-26.

25.Colton HR, Casper JK. Compreendendo os problemas de voz uma perspective fisiológica ao diagnóstico e tratamento. Porto Alegre: Artes Médi- cas; 1996. p. 88-9/292.

26. Máster S, De Biase N, Curcio D, Pontes AAL. Fonação inspiratória e estroboscopia: recursos complementares no diagnóstico colaborativo. In: Behlau M, organizador. O melhor que vi e ouvi: atualização em laringe e voz. Rio de Janeiro: Revinter; 2001. p. 247-53.

27. Corazza VR, Silva VFC, Queija DS, Dedivits RA, Barros APB. Correlação entre os achados estroboscópicos, perceptivo-auditivos e acústicos em adultos sem queixa vocal. Rev Bras Otorrinolaringol. 2004; 70(1):30-4.

28. Pinho SMR. Terapia vocal. In: Pinho SMR. Tópicos em voz. Rio de Janeiro: Guanabara-Koogan; 2001. p. 1-16.

29. Pontes P, Kyrillos L, Behlau M, De Biase N, Pontes A. Vocal nodules and laryngeal morphology. J Voice. 2002; 16(3):408-14.

30. De Biase N, Ponte P, Vieira VP, De Biase S. O modo de coaptação glótica em crianças no diagnóstico diferencial de alteração estrutural mínima. Rev Bras Otorrinolaringol. 2004; 70(4):457-62.

31. Cotes C, Guedes L, Silva A. A relação entre constrições e patologias de pregas vocais. In: Behlau M, organizador. O melhor que vi e ouvi: atualização em laringe e voz. Rio de Janeiro: Revinter; 1998. p. 305-6.

32. Ivano FH, Nassif Filho ACN, Gortz F, Miyake RT, Nakamoto RH. Diagnóstico da doença do refluxo gastroesofágico-endoscopia versus $\mathrm{pH}$ metria de 24 horas. Rev Bras Otorrinolaringol. 2000; 66(5):468-73.

33. Gomesi GF, Macedo Filho ED, Coelho JCU, Salvalaggio PRO, Sebastião APM, Gums SD. Achados histológicos na parede posterior da laringe em pacientes com refluxo gastroesofageano (GERD). Rev Bras Otorrinolaringologia. 2001; 67(6):770-4.

34. Behlau M, Madazio G, Feijó D, Azevedo R, Gielow I, Rehder MI. Aperfeiçoamento vocal e tratamento fonoaudiológico das disfonias. In: Behlau M. Voz: o livro do especialista. v. 2. Rio de Janeiro: Revinter; 2005. p. 410-519.

35. Rosa IG, Bompet R. Singers voice perceptive analysis through tip of the tongue sonorized vibration. In: World voice consortium. 2nd. World Voice Congress and 5th International Symposium of Phonosurgery scientific program. São Paulo; 1999. p.107-8.

36. Guberfain J, Muller MM, Sarvat M. The perceptual, acoustics and laryngological analysis of tip of tongue sound vibration technique and it's consequences in actors. In: World voice consortium. 2nd. World Voice Congress and 5th International Symposium of Phonosurgery scientific program. São Paulo; 1999. p. 55. 
37. Elliot N, Sundberg J, Gramming P. Physiological aspects of a vocal exercise. J Voice. 1997; 11:171-7. 38. Castro LCD, Behlau M. Análise acústica dos efeitos da técnica do "b" prolongado em indivíduos sem queixa vocal. In: Behlau M, Gasparini G, organizador. A voz do especialista. v. 3. Rio de Janeiro: Revinter; 2006. p. 15-21.

39. Krimberg CD, Behlau M. Análise visual e perceptivo-auditiva da técnica finlandesa do "b" prolongado em indivíduos adultos sem queixa vocal. In: Behlau M, Gasparini G, organizador. A voz do especialista. v. 3. Rio de Janeiro: Revinter; 2006. p. 141-58.

40. Kelly CL, Fischer KV. Stroboscopic and acoustic measures of inspiratory phona-tion. J Voice. 1999; 13(3):389-402.

41. Behlau M, Pontes P. Avaliação e tratamento das disfonias. São Paulo: Lovise; 1995. p. 215-8.

RECEBIDO EM: 23/11/2006

ACEITO EM: 23/07/2007

Endereço para correspondência:

Rua Mariano Sendra dos Santos Edifício Aníbal Guedes, 88 ap. 604

Duque de Caxias - RJ

CEP: $25010-080$

Tel: (21) 26716620 / 91825427

E-mail: sgpastana@ig.com.br 